\title{
Association between MEG3/miR-181b polymorphisms and risk of ischemic stroke
}

Xuemei Han ${ }^{1}$, Zhaoshi Zheng ${ }^{1}$, Chunhui Wang ${ }^{2}$ and Libo Wang ${ }^{1 *}$

\begin{abstract}
Background: Recent evidence suggests that long non-coding RNAs (IncRNAs) are key regulators in the pathological process of ischemic stroke (IS). Maternally expressed gene 3 (MEG3) was observed to be up-regulated in IS, acting as a competing endogenous RNA for miR-181b to regulate ischemic brain injury. The purpose of this study was to evaluate the association of tagSNPs in MEG3 (i.e., rs7158663 and rs4081134) and miR-181 b rs322931 with IS risk.

Methods: Genomic DNA was extracted from blood samples of 509 patients with IS and 668 healthy controls. Genotyping of MEG3 rs7158663, rs4081134, and miR-181b rs322931 was performed by TaqMan assay. The transcriptional activity was measured using the Dual-Luciferase Reporter Assay kit.

Results: Single-site analysis revealed a significantly higher risk of IS being associated with miR-181b rs322931 CT and CT/TT genotypes (CT vs. CC: adjusted OR=1.48, 95\% Cl: 1.13-1.95, $P=0.005 ; C T / T$ vs. CC: adjusted OR=1.52, 95\% Cl: 1.17-1.97, $P=0.002$ ). Combined analyses revealed that combined genotypes (rs7158663 GG + rs322931 CT/TT and rs7158663 AG/AA + rs322931 CT/TT) increased IS risk compared to genotypes of rs7158663 GG + rs322931 CC. Stratification analyses showed that patients carrying miR-181b rs322931 CT/TT genotypes had higher levels of low-density lipoprotein cholesterol (LDL_C) $(P=0.01)$. Moreover, results from logistic regression analysis showed that rs322931 CT/TT genotypes were risk factors besides hypertension, total cholesterol, triglyceride, and LDL_C. Further dual-luciferase reporter assay showed that the rs322931 T allele had lower levels of luciferase activity than the rs322931 C allele.
\end{abstract}

Conclusion: These findings indicate that miR-181b rs322931 may singly or jointly contribute to the risk of IS.

Keywords: Long non-coding RNAs, Maternally expressed gene 3, miR-181, Polymorphism, Ischemic stroke

\section{Introduction}

Stroke is the major cause of morbidity and mortality worldwide, with about 15 million new cases and 5 million death each year $[1,2]$. Ischemic stroke (IS), the most common type, occupies $85-90 \%$ of total strokes [3, 4]. China, the most populous country in the world, is facing serious public health implications of IS epidemic [5-7]. Etiologically, IS is a complex disorder, involving in a series of risk factors including hypertension, dyslipidemia, obesity, diabetes, smoking as well as genetic factor [8-11]. Adults with both high triglyceride (TG), low high-density lipoprotein cholesterol (HDL-C), and high low-density lipoprotein cholesterol (LDL-C), particularly those with diabetes, have an increased risk of IS [12]. Supplementation with plant extracts may result in improvement of glycemic and

\footnotetext{
* Correspondence: 935583975@qq.com

${ }^{1}$ No. 1 Department of Neurology, China-Japan Union Hospital of Jilin

University, Changchun, Jilin 130031, People's Republic of China

Full list of author information is available at the end of the article
}

serum lipid levels in patients with dyslipidemia, and finally reduce the risk of IS $[13,14]$. Genetic dissection of IS has targeted many association studies and identified some potential candidate genes such as apolipoprotein A-V, apolipoprotein B, ATP-binding cassette transporter 1, brain-derived neurotrophic factor (BDNF), and arachidonate 5-lipoxygenase-activating protein (ALOX5AP) [15-17]. The selection of these genes was based on important signal pathways in the process of pathophysiology of IS, such as apolipoprotein homeostasis, neurological injury recovery, inflammation and abnormalities in 5-lipoxygenase metabolism [15-17]. Nevertheless, there are several other functional candidate genes that are of great value to be analyzed.

Non-coding RNAs were initially thought to be transcriptional noise without any function. However, it is nowadays believed to play crucial roles in the regulation of information flow from DNA to protein, such as DNA

(C) The Author(s). 2018 Open Access This article is distributed under the terms of the Creative Commons Attribution 4.0 International License (http://creativecommons.org/licenses/by/4.0/), which permits unrestricted use, distribution, and 
replication, RNA splicing, and translation regulation [18-20]. According to the length, non-coding RNAs were classified into microRNAs (miRNAs, 18-24 nucleotides), long non-coding RNAs (lncRNAs, $\geq 200$ nucleotides) and etc. Recently, amounts of non-coding RNAs were found to be aberrantly expressed in IS [21-25]. Among them, maternally expressed gene 3 (MEG3) was observed to be up-regulated in IS, acting as a competing endogenous RNA for miR-181b [26-29]. Knockdown of MEG3 can protect against ischemic damage and improve neurobehavioral outcomes [26, 30]. These findings indicate that the abnormal expression of MEG3 may be a key event in the pathophysiology of IS.

Currently, miRNAs/lncRNAs-related single nucleotide polymorphisms (SNPs) have been demonstrated to be predictors of susceptibility of IS by influencing their expression [31, 32]. In 2016, Cao et al. reported a tagSNP in MEG3 (rs7158663) and the rs7158663 AA genotype had a $96 \%$ increased risk of colorectal cancer [33]. In 2018, Zhuo et al. reported another tagSNP in MEG3 (rs4081134) and carriers with rs4081134 AG/AA genotypes tended to develop neuroblastoma among children $>18$ month of age and clinical stage III/IV disease [34]. Moreover, a genetic variant of rs322931 was reported to influence the expression of miR-181a and miR-181b [35]. To date, no association study was conducted for the 3 SNPs with IS risk. In this study, we instigated the association between the 3 SNPs and IS susceptibility in a Chinese Han population comprising 509 IS patients and 668 age-, gender-, and ethnicity-matched controls. Our purpose was to identify new candidates for the etiology of IS.

\section{Materials and methods}

\section{Study population}

The study population was described in our previous study [36]. Briefly, a total of 509 patients with IS and 668 controls were consecutively recruited from the China-Japan Union Hospital of Jilin University between March 2014 and July 2017. Diagnosis of IS was made according to clinical manifestations and subsequently confirmed by computed tomography scans and magnetic resonance imaging. Patients were excluded if they had hemorrhagic stroke, subarachnoid hemorrhage, traumatic brain injury, malignancy, or other brain inflammatory diseases. IS subtypes were classified according to the criteria of Trial of Org 10,172 in Acute Stroke Treatment as described previously [37]. Controls were healthy subjects from the same hospital during the same time period as the cases. The controls were frequency matched to cases with regard to age, gender, living area, and ethnicity. The exclusion criteria for controls were: i) non-Chinese Han population; ii) having a family history of IS. Diagnosis of hypertension was made according to the criteria: blood pressure $\geq 140 / 90 \mathrm{mmHg}$ on two consecutive occasions at least $24 \mathrm{~h}$ apart or receiving antihypertensive therapy [38]. Diagnosis of diabetes mellitus was made according to the criteria: two fasting glucose $\geq 7.0 \mathrm{mmol} / \mathrm{L}$ and $2 \mathrm{~h}$ postload glucose $\geq 11.1 \mathrm{mmol} / \mathrm{L}$ or receiving treatment with hypoglycemic drugs. Fast serum levels of total cholesterol (TCH), TG, HDL-C, and LDL-C were taken from the hospital database system. The study protocol was approved by the Institutional Ethnical Committee of the China-Japan Union Hospital of Jilin University, and informed consent was signed by each subject. After informed consent was obtained, all subjects contributed 3-5 $\mathrm{ml}$ peripheral venous blood.

\section{SNPs selection}

We selected SNPs according to the following criteria: i) tagSNPs of MEG3; ii) minor allele frequency $>0.10$ in Han Chinese from the 1000 Genome Projects; and iii) in silica analysis predicted that SNPs located in the binding sites of transcription factors. Finally, two MEG polymorphisms of rs7158663 $\mathrm{G}>\mathrm{A}$ and rs4081134 $\mathrm{G}>\mathrm{A}$ were selected for analysis in this study. Moreover, we selected a functional SNP of rs322931 that affects the expression of miR-181a and miR-181b in human brain and blood [35]. The detailed information of the 3 SNPs is described in Fig. 1.

\section{DNA extraction and genotyping}

Genomic DNA was extracted from blood samples using the Genomic DNA Isolation Kit (Tiangen, Beijing, China). SNPs genotyping was performed by TaqMan assay on an ABI 7500 real-time PCR System (Applied Biosystems, Foster City, CA, USA). The probe ID for rs7158663, rs4081134, and rs322931 was C_9693465_10, C_1259786_10, and C_26961572_20, respectively. For quality control, a negative control of distilled water without template was used in each 96-well plate. Furthermore, about 5\% randomly selected samples were reanalyzed by Sanger sequencing, and the results were completely identical.

\section{Dual-luciferase activity assay}

Luciferase activity was detected as previously described [36]. Briefly, 2 fragments were amplified using DNA template from two individuals homozygous for either the rs322931 CC or rs322931 TT genotype. The products were cloned into the pGL3 vector containing a firefly luciferase reporter gene (Promega, Madison, WI, USA). The vectors were transiently transfected into cultured HEK293 cells using the Lipofectamine 3000 (Thermo Fisher Scientific, Waltham, MA, USA), and transcriptional activity was measured using the Dual-Luciferase Reporter Assay kit (Promega) at $48 \mathrm{~h}$ after transfection. The pRL-TK vector with a renilla luciferase reporter gene (Promega) was co-transfected for normalization. The relative luciferase activity was reported as the ratio of firefly to luciferase activity. 


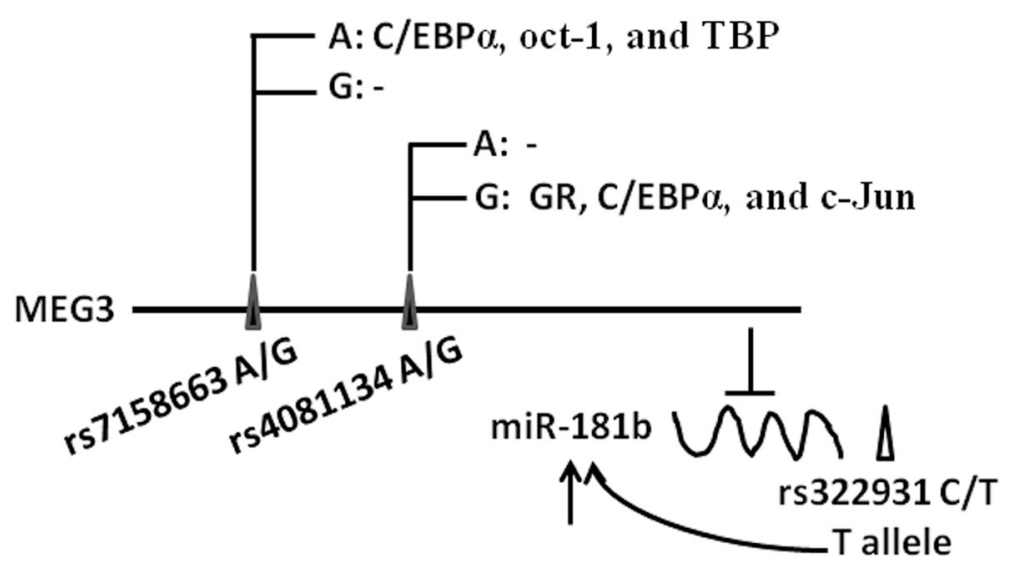

Fig. 1 SNPs related to IncRNA MEG3 and miR-181b. $\Delta$ TagSNPs in MEG3 (i.e., rs7158663 and rs4081134). In silica analysis showed that the rs7158663 A can bind to transcriptional factors [C/EBPa, oct-1, and TATA bingding protein (TBP)] and the rs4081134 G can bind to transcriptional factors [(glucocorticoid receptor GR), C/EBPa, and c-Jun], whereas the rs7158663 G and rs4081134 A can not. $\triangle$ Genome-wide association study indentified a functional polymorphism rs322931. The T allele exhibited higher levels of miR-181b

\section{Statistical analysis}

Statistical data was analyzed using the SPSS software version 19.0 (SPSS, Chicago, IL, USA). Continuous data was presented as mean \pm standard deviation and compared using the Student's $t$-test. Categorical data was presented as number (proportions) and compared using chi-squared test. Hardy-Weinberg equilibrium (HWE) for each SNP in both cases and controls were compared using $X^{2}$ test. The associations of single and combined genotype analyses with IS risk were evaluated using odds ratios (ORs) and 95\% confidence intervals (CIs) after adjustment of age, gender, hypertension, and diabetes mellitus. The linkage disequilibrium (LD) and the association of haplotypes with risk of IS were estimated by the SHEsis software [39]. For multiple comparison, $P$ $<0.017(0.05 / 3)$ was considered as statistically significant after Bonferroni's correction. Multivariable logistic regression analysis was performed to exclude potential confounding variables with age, gender, hypertension, diabetes mellitus, TCH, TG, HDL-C, LDL-C, MEG3 rs7158663, rs4081134 and miR-181b rs322931 entered as covariates in the model. $P$ values less than 0.05 were considered as significant.

\section{Results}

\section{Baseline characteristics of the study population}

The baseline features of the study population are presented in Table 1. The mean age of cases and controls was $59.9 \pm 10.9$ and $58.6 \pm 12.2$, respectively. There were 327 males and 182 females in cases and 414 males and 254 females in controls. No significant difference was observed between the 2 groups for age and gender $(P>$ 0.05). Compared with controls, patients with IS had a higher prevalence of hypertension and diabetes mellitus and higher levels of TCH, TG, and LDL-C.

\section{Association between polymorphisms in MEG3/miR-181b} and IS risk

The genotype frequencies of MEG3 rs7158663, rs4081134 and miR-181b rs322931 were in HWE in both cases and controls $(P>0.05)$. miR-181b rs322931 CT and $\mathrm{CT} / \mathrm{TT}$ genotypes were more frequent in IS patients than in controls ( 33.4 vs. $26.8 \%$ and 38.1 vs. $29.2 \%$ ). A significantly higher risk of IS was associated with rs322931 CT and CT/TT genotypes after adjusting to age, gender, hypertension, and diabetes mellitus (CT vs. CC: adjusted $\mathrm{OR}=1.48,95 \% \mathrm{CI}: 1.13-1.95, P=0.005$; CT/TT vs. CC: adjusted OR $=1.52,95 \% \mathrm{CI}: 1.17-1.97, P$

Table 1 Characteristics of the study population

\begin{tabular}{|c|c|c|c|}
\hline Variables & $\begin{array}{l}\text { Controls, } \\
n=668\end{array}$ & $\begin{array}{l}\text { Patients with IS, } \\
n=509\end{array}$ & $P$ value \\
\hline Age, mean $( \pm S D)$ & $58.6( \pm 12.2)$ & $59.9( \pm 10.9)$ & 0.06 \\
\hline \multicolumn{4}{|l|}{ Gender (\%) } \\
\hline Male & $414(62.0)$ & $327(64.2)$ & \multirow[t]{2}{*}{0.43} \\
\hline Female & $254(38.0)$ & $182(35.8)$ & \\
\hline \multicolumn{4}{|l|}{ Hypertension, n (\%) } \\
\hline Yes & $132(19.8)$ & $280(55.0)$ & \multirow[t]{2}{*}{$<0.001$} \\
\hline No & $536(80.2)$ & $229(45.0)$ & \\
\hline \multicolumn{4}{|c|}{ Diabetes mellitus, n (\%) } \\
\hline Yes & $72(10.8)$ & $80(15.7)$ & \multirow[t]{2}{*}{0.01} \\
\hline No & $596(89.2)$ & $429(84.3)$ & \\
\hline $\mathrm{TCH}, \mathrm{mmol} / \mathrm{L}$ & $4.68 \pm 0.79$ & $5.04 \pm 0.72$ & $<0.001$ \\
\hline $\mathrm{TG}, \mathrm{mmol} / \mathrm{L}$ & $1.11 \pm 0.36$ & $1.84 \pm 1.10$ & $<0.001$ \\
\hline $\mathrm{HDL}-\mathrm{C}, \mathrm{mmol} / \mathrm{L}$ & $1.56 \pm 0.36$ & $1.57 \pm 0.38$ & 0.53 \\
\hline LDL-C, mmol/L & $2.26 \pm 0.97$ & $2.69 \pm 0.98$ & $<0.001$ \\
\hline
\end{tabular}

IS ischemic stroke, SD standard deviation; $\mathrm{TCH}$, total cholesterol, TG triglyceride, $H D L-C$ high-density lipoprotein cholesterol, $L D L-C$ low-density lipoprotein cholesterol 
$=0.002$ ). However, no evidence of association was observed between MEG3 rs7158663 and rs4081134 and IS risk (Table 2).

Stratification analyses were applied to evaluate the association between the 3 polymorphisms and clinical features of IS, such as TCH, TG, HDL-C, and LDL-C. As shown in Table 3, patients carrying miR-181b rs322931 CT/TT genotypes had higher LDL-C levels compared to those carrying $\mathrm{CC}$ genotype $(P=0.01)$. No evident association was found between MEG3 rs7158663 and rs4081134 and clinical features of IS.

The ancestral allele frequencies of the 3 SNPs based on data from the 1000 Genomes Project are summarized in Table 4. Compared with the data in the current study, the frequency of the rs7158663 G allele was significantly lower in Africans $(12.8-32.5 \%$ vs. $73.8 \%$ ) whereas the distributions of the rs4081134 G and rs322931 C were almost similar among the populations.

\section{Combined analysis}

Combined analysis was then performed to estimate the effect of rs7158663 - rs322931 and rs4081134 - rs322931 on IS risk. As shown in Tables 5, 19.3\% of cases and $15.7 \%$ of controls had the combined genotypes of rs7158663 GG and rs322931 CT/TT, and carriers with these loci had a 1.62-fold increased risk of IS compared to carriers with the genotypes of rs7158663 GG and rs322931 CC (95\% CI: 1.15-2.27, $P=0.005$ ).
Furthermore, $18.9 \%$ of cases and $13.5 \%$ of controls had the combined genotypes of rs7158663 AG/AA and rs322931 CT/TT, and carriers with these loci had a 1.85-fold increased risk of IS compared to carriers with the genotypes of rs7158663 GG and rs322931 CC (95\% CI: $1.30-2.62, P=0.001)$. Nevertheless, the distributions of combined genotypes of rs 4081134 and rs322931 did not vary between cases and controls.

\section{Haplotype analysis}

Despite the absence of an LD among the 3 SNPs, the AGC and GGT haplotypes showed an increased risk of IS compared to the GGC haplotype (AGC vs. GGC: $\mathrm{OR}=1.51,95 \%$ CI: $1.20-1.90, P<0.001$; GGT vs. $\mathrm{GGC}: \mathrm{OR}=1.55,95 \% \mathrm{CI}: 1.16-2.07, \quad P=0.003)$ (Table 6).

\section{Multivariate regression analysis}

Multivariate logistic regression analysis initially included the following variables: age, gender, hypertension, diabetes mellitus, TCH, TG, HDL-C, LDL-C, MEG3 rs7158663, rs4081134 and miR-181b rs322931. After stepwise testing, independent factors of IS were identified, including hypertension $(\mathrm{OR}=4.97,95 \% \mathrm{CI}: 3.67-$ 6.73, $P<0.001)$, TCH (OR = 1.24, 95\%CI: $1.02-1.49, P=$ $0.03)$, TG $(\mathrm{OR}=5.89,95 \% \mathrm{CI}: 4.43-7.83, P<0.001)$, LDL-C $(\mathrm{OR}=1.90,95 \% \mathrm{CI}: 1.61-2.24, P<0.001)$, and rs322931 CT/TT genotypes $(\mathrm{OR}=1.41,95 \% \mathrm{CI}: 1.04-$ 1.91, $P=0.03$ ) (Table 7).

Table 2 Association between polymorphisms in MEG3/miR-181b and risk of ischemic stroke

\begin{tabular}{|c|c|c|c|c|c|c|}
\hline Polymorphism & Controls, $n=668(\%)$ & $I S, n=509(\%)$ & Crude OR $(95 \%$ Cl) & $P$ value & Adjusted OR(95\% Cl ${ }^{\dagger}$ & Adjusted $P$ value ${ }^{\dagger}$ \\
\hline \multicolumn{7}{|l|}{ MEG3 rs7158663 } \\
\hline GG & $372(55.7)$ & $252(49.5)$ & 1.00 & & 1.00 & \\
\hline$A G$ & $242(36.2)$ & $202(39.7)$ & $1.23(0.96-1.58)$ & 0.10 & $1.19(0.92-1.55)$ & 0.19 \\
\hline AA & $54(8.1)$ & $55(10.8)$ & $1.50(1.00-2.26)$ & 0.05 & $1.49(0.96-2.32)$ & 0.08 \\
\hline Dominant model & $296(44.3)$ & $257(50.5)$ & $1.28(1.02-1.62)$ & 0.04 & $1.25(0.98-1.60)$ & 0.08 \\
\hline Recessive model & $614(91.9)$ & $454(89.2)$ & $1.38(0.93-2.04)$ & 0.11 & $1.39(0.91-2.12)$ & 0.13 \\
\hline \multicolumn{7}{|l|}{ MEG3 rs4081134 } \\
\hline GG & $392(58.7)$ & $310(60.9)$ & 1.00 & & 1.00 & \\
\hline$A G$ & $230(34.4)$ & $167(32.8)$ & $0.92(0.72-1.18)$ & 0.50 & $0.87(0.67-1.14)$ & 0.31 \\
\hline $\mathrm{AA}$ & $46(6.9)$ & $32(6.3)$ & $0.88(0.55-1.41)$ & 0.60 & $0.82(0.49-1.37)$ & 0.44 \\
\hline Dominant model & $276(41.3)$ & $199(39.1)$ & $0.91(0.72-1.15)$ & 0.44 & $0.86(0.67-1.11)$ & 0.25 \\
\hline Recessive model & $622(93.1)$ & $477(93.7)$ & $0.91(0.57-1.45)$ & 0.68 & $0.88(0.53-1.45)$ & 0.61 \\
\hline \multicolumn{7}{|l|}{ miR-181b rs322931 } \\
\hline $\mathrm{CC}$ & $473(70.8)$ & $315(61.9)$ & 1.00 & & 1.00 & \\
\hline CT & $179(26.8)$ & $170(33.4)$ & $1.43(1.11-1.84)$ & 0.006 & $1.48(1.13-1.95)$ & 0.005 \\
\hline$\pi$ & $16(2.4)$ & $24(4.7)$ & $2.25(1.18-4.31)$ & 0.01 & $1.84(0.91-3.70)$ & 0.09 \\
\hline Dominant model & $195(29.2)$ & $194(38.1)$ & $1.49(1.17-1.91)$ & 0.001 & $1.52(1.17-1.97)$ & 0.002 \\
\hline Recessive model & $652(97.6)$ & $485(95.3)$ & $2.02(1.06-3.84)$ & 0.03 & $1.64(0.82-3.27)$ & 0.16 \\
\hline
\end{tabular}

IS ischemic stroke, $\mathrm{OR}$ odds ratio, $\mathrm{Cl}$ confidence interval. ${ }^{\dagger}$ Adjusted by age, gender, hypertension, and diabetes mellitus 
Table 3 Stratification analyses of polymorphisms in MEG3/miR-181b and clinical features of IS

\begin{tabular}{|c|c|c|c|c|c|c|c|c|}
\hline \multirow[t]{2}{*}{ Genotypes } & \multicolumn{2}{|c|}{$\mathrm{TCH}(\mathrm{mmol} / \mathrm{L})$} & \multicolumn{2}{|l|}{$\mathrm{TG}(\mathrm{mmol} / \mathrm{L})$} & \multicolumn{2}{|c|}{$\mathrm{HDL}-\mathrm{C}(\mathrm{mmol} / \mathrm{L})$} & \multicolumn{2}{|c|}{ LDL-C (mmol/L) } \\
\hline & Mean \pm SD & $P$ value & Mean \pm SD & $P$ value & Mean \pm SD & $P$ value & Mean \pm SD & $P$ value \\
\hline \multicolumn{9}{|c|}{ MEG3 rs7158663 } \\
\hline GG & $5.07 \pm 0.72$ & & $1.82 \pm 1.08$ & & $1.56 \pm 0.38$ & & $2.74 \pm 0.98$ & \\
\hline $\mathrm{AG} / \mathrm{AA}$ & $5.02 \pm 0.72$ & 0.36 & $1.87 \pm 1.12$ & 0.63 & $1.58 \pm 0.39$ & 0.54 & $2.63 \pm 0.97$ & 0.18 \\
\hline \multicolumn{9}{|c|}{ MEG3 rs4081134 } \\
\hline GG & $5.07 \pm 0.66$ & & $1.84 \pm 1.06$ & & $1.58 \pm 0.39$ & & $2.71 \pm 0.93$ & \\
\hline $\mathrm{AG} / \mathrm{AA}$ & $5.00 \pm 0.80$ & 0.31 & $1.86 \pm 1.16$ & 0.81 & $1.55 \pm 0.37$ & 0.36 & $2.65 \pm 1.05$ & 0.49 \\
\hline \multicolumn{9}{|c|}{ miR-181b rs322931 } \\
\hline $\mathrm{CC}$ & $5.01 \pm 0.71$ & & $1.85 \pm 1.09$ & & $1.58 \pm 0.37$ & & $2.60 \pm 0.96$ & \\
\hline $\mathrm{CT} / \mathrm{TT}$ & $5.10 \pm 0.72$ & 0.17 & $1.84 \pm 1.12$ & 0.92 & $1.56 \pm 0.40$ & 0.73 & $2.82 \pm 1.00$ & 0.01 \\
\hline
\end{tabular}

Effect of the rs322931 on transcriptional activity

To elucidate the effect of the rs322931 on transcriptional activity, we performed a luciferase reporter assay and found that HEK293 cells transfected with the rs322931 $\mathrm{T}$ allele had lower levels of luciferase activity than cells transfected with the rs322931 C allele $(P<0.01)$ (Fig. 2), suggesting that decreased transcriptional activity mediated the risk effect of the rs322931 T allele.

\section{Discussion}

In the current study, we aimed to investigate the association of genetic variants in MEG3/miR-181b with susceptibility to IS. We found a significant difference in

Table 4 The ancestral allele frequencies of the 3 SNPs based on data from the 1000 Genomes Project

\begin{tabular}{llll}
\hline Population & $\begin{array}{lll}\text { rs7158663 } \\
\text { G, } \%\end{array}$ & $\begin{array}{l}\text { rs4081134 } \\
\text { G, \% }\end{array}$ & $\begin{array}{l}\text { rs322931 } \\
\text { C, \% }\end{array}$ \\
\hline HapMap-CEU & 50.9 & 62.6 & 81.7 \\
HapMap-HCB & 74.4 & 77.9 & 84.9 \\
HapMap-JPT & 66.9 & 80.8 & 83.1 \\
HapMap-YRI & 12.8 & 77.9 & 84.8 \\
HAPMAP-ASW & 23.5 & 80.6 & 81.6 \\
HAPMAP-CHB & 74.4 & 78.1 & 84.2 \\
HAPMAP-CHD & 81.8 & 72.4 & 84.9 \\
HAPMAP-GIH & 69.9 & 63.6 & 79.0 \\
HAPMAP-LWK & 25.0 & 80.6 & 80.9 \\
HAPMAP-MEX & 59.0 & 74.0 & 65.3 \\
HAPMAP-MKK & 32.5 & 79.0 & 80.7 \\
HAPMAP-TSI & 52.8 & 65.3 & 88.1 \\
Data in the current study & 73.8 & 75.9 & 84.2
\end{tabular}

CEU, Utah residents with Northern and Western European ancestry from the CEPH collection, HCB Han Chinese in Beijing, China, JPT Japanese in Tokyo, Japan, YRI Yoruba in Ibadan, Nigeria, ASW African ancestry in Southwest USA CHB Han Chinese in Beijing, China, CHD Chinese in Metropolitan Denver, Colorado, GIH Gujarati Indians in Houston, Texas, LWK Luhya in Webuye, Kenya, MEX Mexican ancestry in Los Angeles, California, MKK Maasai in Kinyawa, Kenya, TSI Toscans in Italy genotypic distribution of miR-181b rs322931 and IS. Compared to miR-181b rs322931 TT genotype, CT and $\mathrm{CT} / \mathrm{TT}$ genotypes were found to confer 1.48 - and 1.52 -fold increased risks of IS. Stratification analyses showed that patients carrying miR-181b rs322931 CT/ TT genotypes had higher LDL-C levels. Although we did not observe any significant association between MEG3 rs7158663 and IS, the combined genotypes of rs7158663 GG and rs322931 CT/TT were associated with a $62 \%$ increased risk of IS. Multivariate logistic regression analysis identified that rs322931 CT/TT genotype was a risk factor for IS in addition to hypertension, TCH, TG, and LDL-C. Further dual-luciferase reporter assay showed that the rs322931 $\mathrm{T}$ allele had lower levels of luciferase activity than the rs322931 C allele. Our results indicate that miR-181b rs322931 may singly or jointly contribute to the risk of IS in the Chinese population.

Previous association studies focused on SNPs in the protein-coding genes and the risk of IS [16, 17]. For example, Bao et al. reported an SNP rs6265 AA genotype in $B D N F$ had a 0.57 -fold decreased risk of IS [16]. Chen et al. reported another SNP ALOX5AP SG13S114 A allele had a 0.86-fold decreased risk of IS [17]. Recently, the role of non-coding RNAs in the pathogenesis of IS attracted much attention, with differentially expressed lncRNAs in both human and animal model of IS [22, 23]. Moreover, lncRNA-related SNPs were reported to be potential genetic markers and therapeutic targets of IS. Wang et al. investigated 6 tagSNPs in lncRNA H19 and found that rs217727 TT and rs4929984 AA genotype increased IS risk, with an adjusted OR of 4.29 and 3.02, respectively [40]. The findings indicate that lncRNAs play fundamental roles in the development of IS.

MEG3, located on chromosome 14q32.3 in human genome, was firstly identified as a lncRNA with function of tumor suppressor [41]. Subsequent results revealed that MEG3 is implicated in biological processes of IS 
Table 5 Combined analyses of polymorphisms in MEG3/miR-181b with IS risk

\begin{tabular}{llll}
\hline Combined genotypes & Controls, $n=668(\%)$ & IS, $n=509(\%)$ & OR (95\% Cl) \\
\hline MEG3 rs7158663- miR-181b rs322931 & & & $P$ value \\
rs7158663 GG + rs322931 CC & $267(40.0)$ & $154(30.3)$ & 1.00 \\
rs7158663 GG + rs322931 CT/TT & $105(15.7)$ & $98(19.3)$ & $1.62(1.15-2.27)$ \\
rs7158663 AG/AA + rs322931 CC & $206(30.8)$ & $161(31.6)$ & $1.36(1.02-1.80)$ \\
rs7158663 AG/AA + rs322931 CT/TT & $90(13.5)$ & $96(18.9)$ & $1.85(1.30-2.62)$ \\
MEG3 rs4081134- miR-181b rs322931 & & & 0.005 \\
rs4081134 GG + rs322931 CC & $281(42.1)$ & $203(39.9)$ & 1.00 \\
rs4081134 GG + rs322931 CT/TT & $111(16.6)$ & $107(21.0)$ & $1.33(0.97-1.84)$ \\
rs4081134 AG/AA + rs322931 CC & $192(28.7)$ & $112(22.0)$ & $0.81(0.60-1.08)$ \\
rs4081134 AG/AA + rs322931 CT/TT & $84(12.6)$ & $87(17.1)$ & $1.43(1.01-2.03)$ \\
\hline
\end{tabular}

IS ischemic stroke, OR Odds ratio, $\mathrm{Cl}$ confidence interval

[26-28, 30]. Altered expression of cerebral MEG3 was observed both in vitro and in vivo, activating p53 to mediate ischemic neuronal death in stroke [27, 28]. Downregulation of MEG3 can protect against ischemic damage and enhance neurobehavioral outcomes $[26,30]$. Furthermore, MEG3 functions as a competing endogenous RNA for miR-181b to regulate 12/15-LOX expression in middle cerebral artery occlusion-induced ischemic infarct of brain nerve cells [29]. In 2016, an association study including 518 cases and 527 controls was conducted by Cao and colleagues who genotyped 5 tagSNPs in MEG3 and found that rs7158663 AA genotype but not other SNPs increased the risk of colorectal cancer [33]. In 2018, Zhou et al. genotyped 2 tagSNPs in MEG3 in 392 neuroblastoma children and 783 controls and found that subjects carrying rs4081134 AG/AA genotypes tended to develop neuroblastoma among subgroups with age $>18$ month and clinical stage III + IV disease, with an adjusted OR of 1.36 and 1.47, respectively [34]. To date, we are not aware of any association study of SNPs in MEG3 in IS. In this study, we explored for the first time the association between 2 tagSNPs (i.e., rs7158663 and rs4081134) and IS risk in 509 IS patients and 668 controls. Although we failed to find any association of the 2 SNPs with IS risk in

Table 6 Haplotype analysis of the MEG3/miR-181b polymorphisms between cases and controls

\begin{tabular}{lllll}
\hline Haplotype $^{\text {a }}$ & Controls, N (\%) & IS, N (\%) & OR (95\% Cl) & $P$ value \\
\hline GGC & $653(48.9)$ & $429(42.1)$ & 1.00 & \\
AGC & $206(15.4)$ & $204(20.0)$ & $1.51(1.20-1.90)$ & $<0.001$ \\
GAC & $180(13.5)$ & $127(12.5)$ & $1.07(0.83-1.39)$ & 0.59 \\
GGT & $111(8.3)$ & $113(11.1)$ & $1.55(1.16-2.07)$ & 0.003 \\
AAC & $85(6.4)$ & $40(3.9)$ & $0.72(0.48-1.06)$ & 0.10 \\
AGT & $44(3.3)$ & $41(4.0)$ & $1.42(0.91-2.21)$ & 0.12 \\
GAT & $41(3.1)$ & $37(3.6)$ & $1.37(0.87-2.18)$ & 0.18 \\
\hline
\end{tabular}

${ }^{\mathrm{a}}$ Only the frequency $>3 \%$ was presented single-site analysis, the combined genotypes of MEG3 rs7158663 and miR-181b rs322931 conferred the susceptibility of IS, further supporting the idea that MEG3 and miR-181b have a crosstalk in the pathophysiology of IS [29].

miR-181b, a kind of miRNAs, was demonstrated to be a key player in the regulation of circulating low-density lipoprotein cholesterol levels [42] and neural ischemic injuries [43-47]. In mouse brain following middle cerebral artery occlusion and oxygen-glucose deprivation-treated cells, miR-181b was observed to be downregulated, and the downregulation protects against ischemic injury via targeting heat shock protein A5, ubiquitin carboxyl-terminal hydrolase isozyme $\mathrm{L} 1$ and cylindromatosis [43, 47]. Administration of miR-181b can not only modulate macrophage polarization and attenuate atherosclerotic plaque vulnerability but also increase M2 markers in macrophage cells through directly targeting Notch1 [44]. Exosomes-derived miR-181b-5p can accelerate angiogenesis of ischemic injury both in vivo and in vitro by targeting transient receptor potential melastatin 7 [45]. Moreover, electroacupuncture can enhance rehabilitation against stroke by targeting miR-181b/PirB/ RhoA/GAP43 axis and leading to epigenetic changes [46]. These findings strongly suggest that miR-181b may be a potential therapeutic target for IS. No study however investigated the

Table 7 Logistic regression analysis for independent risk factors of ischemic stroke

\begin{tabular}{lllll}
\hline Variables & B & Walds & OR $(95 \%$ Cl) & $P$ value \\
\hline Hypertension & 1.60 & 107.13 & $4.97(3.67-6.73)$ & $<0.001$ \\
TCH & 0.21 & 4.70 & $1.24(1.02-1.49)$ & 0.03 \\
TG & 1.77 & 148.89 & $5.89(4.43-7.83)$ & $<0.001$ \\
LDL-C & 0.64 & 59.63 & $1.90(1.61-2.24)$ & $<0.001$ \\
rs322931 CT/TT & 0.34 & 4.82 & $1.41(1.04-1.91)$ & 0.03 \\
\hline
\end{tabular}

$O R$ odds ratio, $\mathrm{Cl}$ confidence interval, $T C H$ total cholesterol, $T G$ triglyceride, $L D L-C$ low-density lipoprotein cholesterol 


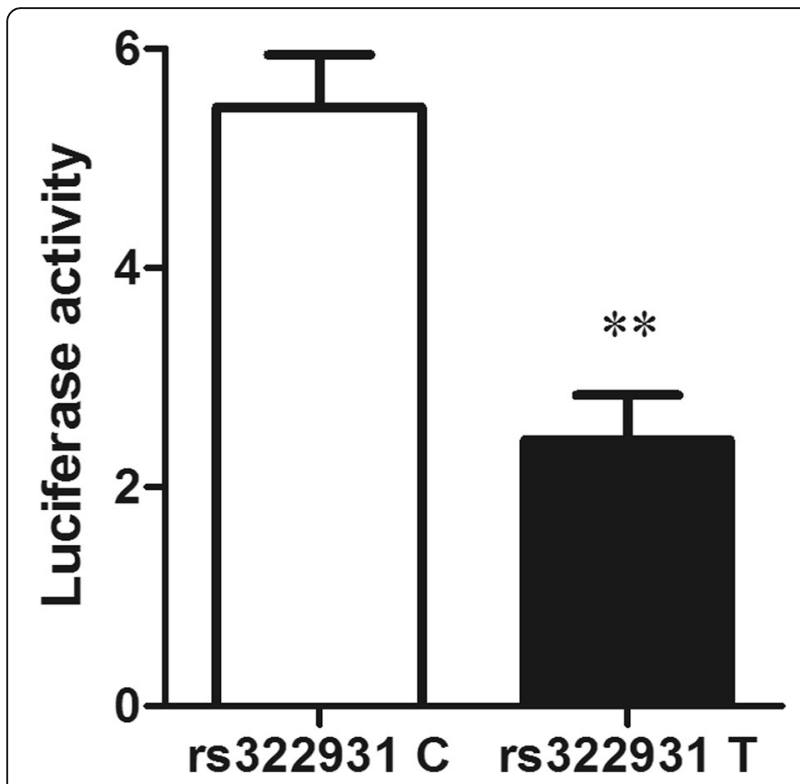

Fig. 2 Effect of the rs322931 on transcriptional activity. The fragment containing the rs322931 C or rs322931 T allele was transfected into HEK293 cells. pGL3-SV40 was used as an internal control and relative luciferase activity was measured $48 \mathrm{~h}$ post-transfection. Data are expressed as mean \pm standard error $\left({ }^{* *} P<0.01\right)$

association of miR-181b related SNP with IS risk. In this study, we hypothesized that rs322931 may contribute to the risk of IS since the polymorphism can influence the expression of miR-181b [35]. Our findings for the first time confirmed this hypothesis. We found that miR-181b rs322931 CT/TT was an independent risk factor in both single-site association analysis and logistic regression analysis. The risk effect may partly be caused by lower levels of transcriptional activity.

Some limitations in this study should be noted. The study is based on hospitalized cases and controls, and thus the selection bias cannot be ruled out. Considering some demographic and clinical factors influencing genetic predisposition of IS, it would be interesting to perform gene-environment interaction analysis. However, lack of comprehensive data, such as drinking habits and tobacco smoking makes it unfeasible to run such an analysis. We, therefore, encourage further investigation of MEG3 rs7158663, rs4081134 and miR-181b rs322931 in population-based studies and gene-environment interaction analysis, which may reveal reliable models of the SNPs in IS occurrence.

\section{Conclusion}

To our knowledge, this is the first study analyzing genetic polymorphisms in MEG3/miR-181b with IS to date, and it indicates a significant association between miR-181b rs322931 and the risk of IS, partly caused by reducing transcriptional activity. Additionally, this study adds novel information about combined genotypes (rs7158663 GG + rs322931 CT/TT and rs7158663 AG/AA + rs322931 $\mathrm{CT} / \mathrm{TT}$ ) increasing IS risk and rs322931 CT/TT genotype being an independent risk factor for IS. Further investigation is required regarding how the polymorphism influences IS risk.

\section{Abbreviations}

ALOX5AP: Arachidonate 5-lipoxygenase-activating protein; ASW: African ancestry in Southwest USA; BDNF: Brain-derived neurotrophic factor; CEU: Utah residents with Northern and Western European ancestry from the CEPH collection; CHB: Han Chinese in Beijing, China; CHD: Chinese in Metropolitan Denver, Colorado; Cls: Confidence intervals; GIH: Gujarati Indians in Houston, Texas; HCB: Han Chinese in Beijing, China; HDL-C: High-density lipoprotein cholesterol; HWE: Hardy Weinberg equilibrium; IS: Ischemic stroke; JPT: Japanese in Tokyo, Japan; LD: Linkage disequilibrium; LDL-C: low-density lipoprotein cholesterol; IncRNAs: Long non-coding RNAs; LWK: Luhya in Webuye, Kenya; MEG3: Maternally expressed gene 3; MEX: Mexican ancestry in Los Angeles, California; miRNAs: microRNAs; MKK: Maasai in Kinyawa, Kenya; OR: odds ratio; SNPs: single nucleotide polymorphisms; TBP: TATA bingding proteinGRGlucocorticoid receptor; TCH: Total cholesterol; TG: Triglyceride; TSI: Toscans in Italy; YRI: Yoruba in Ibadan, Nigeria

\section{Acknowledgements}

We would like to thank all participants who agreed to participate in the study.

\section{Funding}

None.

\section{Availability of data and materials}

The datasets supporting the conclusions of this article are included within the article.

\section{Authors' contributions}

WL designed and wrote the manuscript. HX and ZZ performed experiments and statistical analysis. HX and WC collected samples. All authors read and approved the final manuscript

\section{Ethics approval and consent to participate}

The study was approved by the Institutional Review Board of the China-Japan Union Hospital of Jilin University. All patients agreed to participate in the study and signed the informed consent.

\section{Consent for publication}

Not applicable.

\section{Competing interests}

The authors declare that they have no competing interests.

\section{Publisher's Note}

Springer Nature remains neutral with regard to jurisdictional claims in published maps and institutional affiliations.

\section{Author details}

${ }^{1}$ No. 1 Department of Neurology, China-Japan Union Hospital of Jilin University, Changchun, Jilin 130031, People's Republic of China. ${ }^{2}$ Department of Neurosurgery, the Hospital of Jilin Province, Changchun, Jilin 130031, People's Republic of China.

Received: 19 May 2018 Accepted: 4 December 2018 Published online: 22 December 2018

\section{References}

1. Bonita R, Mendis S, Truelsen T, Bogousslavsky J, Toole J, Yatsu F. The global stroke initiative. Lancet Neurol. 2004;3:391-3.

2. Donnan GA, Fisher M, Macleod M, Davis SM. Stroke. Lancet. 2008:371:1612-23.

3. Adams HP Jr, Del Zoppo G, Alberts MJ, Bhatt DL, Brass L, Furlan A, Grubb RL, Higashida RT, Jauch EC, Kidwell C, et al. Guidelines for the early management of adults with ischemic stroke: a guideline from the American 
Heart Association/American Stroke Association Stroke Council, Clinical Cardiology Council, Cardiovascular Radiology and Intervention Council, and the Atherosclerotic Peripheral Vascular Disease and Quality of Care Outcomes in Research Interdisciplinary Working Groups: the American Academy of Neurology affirms the value of this guideline as an educational tool for neurologists. Stroke. 2007;38:1655-711.

4. Feigin $\mathrm{VL}$, Forouzanfar MH, Krishnamurthi R, Mensah GA, Connor M, Bennett DA, Moran AE, Sacco RL, Anderson L, Truelsen T, et al. Global and regional burden of stroke during 1990-2010: findings from the global burden of disease study 2010. Lancet. 2014;383:245-54.

5. Kong D, Liu X, Lian H, Zhao X, Zhao Y, Xu Q, Peng B, Wang H, Fang Q Zhang $S$, et al. Analysis of hospital charges of inpatients with acute ischemic stroke in Beijing, China, 2012-2015. Neuroepidemiology. 2018;50:63-73.

6. Zhang N, Liu G, Zhang G, Fang J, Wang Y, Zhao X, Pan Y, Guo L, Wang Y. China National Stroke Registry I: external validation of the iScore for predicting ischemic stroke mortality in patients in China. Stroke. 2013:44:1924-9.

7. Zhao JJ, He GQ, Gong SY, He L. Status and costs of primary prevention for ischemic stroke in China. J Clin Neurosci. 2013;20:1427-32.

8. Yong H, Foody J, Linong J, Dong Z, Wang Y, Ma L, Meng HJ, Shiff S, Dayi H. A systematic literature review of risk factors for stroke in China. Cardiol Rev. 2013;21:77-93

9. Bak S, Gaist D, Sindrup SH, Skytthe A, Christensen K. Genetic liability in stroke: a long-term follow-up study of Danish twins. Stroke. 2002;33:769-74.

10. Dichgans M. Genetics of ischaemic stroke. Lancet Neurol. 2007;6:149-61.

11. Schulz UG, Flossmann E, Rothwell PM. Heritability of ischemic stroke in relation to age, vascular risk factors, and subtypes of incident stroke in population-based studies. Stroke. 2004;35:819-24.

12. Lee JS, Chang PY, Zhang Y, Kizer JR, Best LG, Howard BV. Triglyceride and HDL-C dyslipidemia and risks of coronary heart disease and ischemic stroke by glycemic Dysregulation status: the strong heart study. Diabetes Care. 2017:40:529-37.

13. Hajmohammadi Z, Heydari M, Nimrouzi M, Faridi P, Zibaeenezhad MJ, Omrani GR, Shams M. Rhus coriaria L. increases serum apolipoprotein-A and high-density lipoprotein cholesterol levels: a double-blind placebocontrolled randomized clinical trial. J Integr Med. 2018;16:45-50.

14. Samadi N, Mozaffari-Khosravi H, Rahmanian M, Askarishahi M. Effects of bee propolis supplementation on glycemic control, lipid profile and insulin resistance indices in patients with type 2 diabetes: a randomized, doubleblind clinical trial. J Integr Med. 2017;15:124-34.

15. Au A, Griffiths LR, Irene L, Kooi CW, Wei LK. The impact of APOA5, APOB, $A P O C 3$ and $A B C A 1$ gene polymorphisms on ischemic stroke: evidence from a meta-analysis. Atherosclerosis. 2017;265:60-70

16. Bao MH, Zhu SZ, Gao XZ, Sun HS, Feng ZP. Meta-analysis on the association between brain-derived Neurotrophic factor polymorphism rs6265 and ischemic stroke, Poststroke depression. J Stroke Cerebrovasc Dis. 2018;27:1599-608.

17. Chen Z, Zheng J, Liu W, Yang K, Li K, Huang B, Zhu R, Lu X, Li L. The SG13S114 polymorphism of the ALOX5AP gene is associated with ischemic stroke in Europeans: a meta-analysis of 8062 subjects. Neurol Sci. 2017;38: 579-87

18. Jeffares DC, Poole AM, Penny D. Relics from the RNA world. J Mol Evol. 1998:46:18-36.

19. Poole AM, Jeffares DC, Penny D. The path from the RNA world. J Mol Evol. 1998:46:1-17.

20. Poole A, Jeffares D, Penny D. Early evolution: prokaryotes, the new kids on the block. Bioessays. 1999;21:880-9.

21. He W, Wei D, Cai, Chen S, Li S, Chen W. Altered long non-coding RNA Transcriptomic profiles in ischemic stroke. Hum Gene Ther. 2018.

22. Dykstra-Aiello C, Jickling GC, Ander BP, Shroff N, Zhan X, Liu D, Hull H, Orantia M, Stamova BS, Sharp FR. Altered expression of long noncoding RNAs in blood after ischemic stroke and proximity to putative stroke risk loci. Stroke. 2016;47:2896-903.

23. Liu C, Yang J, Zhang C, Liu M, Geng X, Ji X, Du H, Zhao H. Analysis of long non-coding RNA expression profiles following focal cerebral ischemia in mice. Neurosci Lett. 2018;665:123-9.

24. Tan KS, Armugam A, Sepramaniam S, Lim KY, Setyowati KD, Wang CW, Jeyaseelan K. Expression profile of MicroRNAs in young stroke patients. PLoS One. 2009:4:e7689.

25. Sorensen SS, Nygaard AB, Nielsen MY, Jensen K, Christensen T. miRNA expression profiles in cerebrospinal fluid and blood of patients with acute ischemic stroke. Transl Stroke Res. 2014;5:711-8.

26. Yan H, Rao J, Yuan J, Gao L, Huang W, Zhao L, Ren J. Long non-coding RNA MEG3 functions as a competing endogenous RNA to regulate ischemic neuronal death by targeting miR-21/PDCD4 signaling pathway. Cell Death Dis. 2017:8:3211.

27. Yan H, Yuan J, Gao L, Rao J, Hu J. Long noncoding RNA MEG3 activation of p53 mediates ischemic neuronal death in stroke. Neuroscience. 2016:337:191-9.

28. Bao MH, Szeto V, Yang BB, Zhu SZ, Sun HS, Feng ZP. Long non-coding RNAs in ischemic stroke. Cell Death Dis. 2018:9:281.

29. Liu X, Hou L, Huang W, Gao Y, Lv X, Tang J. The mechanism of long noncoding RNA MEG3 for neurons apoptosis caused by hypoxia: mediated by miR-181b-12/15-LOX signaling pathway. Front Cell Neurosci. 2016;10:201.

30. Liu J, Li Q, Zhang KS, Hu B, Niu X, Zhou SM, Li SG, Luo YP, Wang Y, Deng ZF. Downregulation of the long non-coding RNA Meg3 promotes angiogenesis after ischemic brain injury by activating notch signaling. Mol Neurobiol. 2017:54:8179-90.

31. Xiang Y, Guo J, Peng YF, Tan T, Huang HT, Luo HC, Wei YS. Association of miR-21, miR-126 and miR-605 gene polymorphisms with ischemic stroke risk. Oncotarget. 2017:8:95755-63.

32. Wang P, He Q, Liu C, He SZ, Zhu SY, Li YW, Su W, Xiang ST, Zhao B. Functional polymorphism rs3783553 in the $3^{\prime}$-untranslated region of IL-1A increased the risk of ischemic stroke: a case-control study. Medicine (Baltimore). 2017;96:e8522.

33. Cao X, Zhuang S, Hu Y, Xi L, Deng L, Sheng H, Shen W. Associations between polymorphisms of long non-coding RNA MEG3 and risk of colorectal cancer in Chinese. Oncotarget. 2016;7:19054-9.

34. Zhuo ZJ, Zhang R, Zhang J, Zhu J, Yang T, Zou Y, He J, Xia H. Associations between IncRNA MEG3 polymorphisms and neuroblastoma risk in Chinese children. Aging (Albany NY). 2018:10:481-91.

35. Wingo AP, Almli LM, Stevens JS, Jovanovic T, Wingo TS, Tharp G, Li Y, Lori A, Briscione $M$, Jin $P$, et al. Genome-wide association study of positive emotion identifies a genetic variant and a role for microRNAs. Mol Psychiatry. 2017; 22:774-83.

36. Zheng Z, Liu S, Wang C, Han X. A functional polymorphism rs145204276 in the promoter of long noncoding RNA GAS5 is associated with an increased risk of ischemic stroke. J Stroke Cerebrovasc Dis. 2018;27:3535-41.

37. Adams HP Jr, Bendixen BH, Kappelle LJ, Biller J, Love BB, Gordon DL, Marsh EE 3rd. Classification of subtype of acute ischemic stroke. Definitions for use in a multicenter clinical trial. TOAST. Trial of Org 10172 in Acute Stroke Treatment. Stroke. 1993:24:35-41.

38. Chobanian AV, Bakris GL, Black HR, Cushman WC, Green LA, Izzo JL Jr, Jones DW, Materson BJ, Oparil S, Wright JT Jr, et al. Seventh report of the joint National Committee on prevention, detection, evaluation, and treatment of high blood pressure. Hypertension. 2003;42:1206-52.

39. Shi YY, He L. SHEsis, a powerful software platform for analyses of linkage disequilibrium, haplotype construction, and genetic association at polymorphism loci. Cell Res. 2005;15:97-8.

40. Wang J, Cao B, Han D, Sun M, Feng J. Long non-coding RNA H19 induces cerebral ischemia reperfusion injury via activation of autophagy. Aging Dis. 2017:8:71-84.

41. Zhou $Y$, Zhang $X$, Klibanski A. MEG3 noncoding RNA: a tumor suppressor. J Mol Endocrinol. 2012;48:R45-53.

42. Coffey AR, Smallwood TL, Albright J, Hua K, Kanke M, Pomp D, Bennett BJ, Sethupathy P. Systems genetics identifies a co-regulated module of liver microRNAs associated with plasma LDL cholesterol in murine diet-induced dyslipidemia. Physiol Genomics. 2017;49:618-29.

43. Peng Z, Li J, Li Y, Yang X, Feng S, Han S, Li J. Downregulation of miR-181b in mouse brain following ischemic stroke induces neuroprotection against ischemic injury through targeting heat shock protein A5 and ubiquitin carboxyl-terminal hydrolase isozyme L1. J Neurosci Res. 2013;91:1349-62.

44. An TH, He QW, Xia YP, Chen SC, Baral S, Mao L, Jin HJ, Li YN, Wang MD, Chen JG, et al. MiR-181b antagonizes atherosclerotic plaque vulnerability through modulating macrophage polarization by directly targeting Notch1. Mol Neurobiol. 2017:54:6329-41.

45. Yang Y, Cai Y, Zhang Y, Liu J, Xu Z. Exosomes secreted by adipose-derived stem cells contribute to angiogenesis of brain microvascular endothelial cells following oxygen-glucose deprivation in vitro through MicroRNA181b/TRPM7 Axis. J Mol Neurosci. 2018.

46. Deng B, Bai F, Zhou H, Zhou D, Ma Z, Xiong L, Wang Q. Electroacupuncture enhances rehabilitation through miR-181b targeting PirB after ischemic stroke. Sci Rep. 2016;6:38997.

47. Zhang H, Zhong K, Lu M, Mei Y, Tan E, Sun X, Tan W. Neuroprotective effects of isosteviol sodium through increasing CYLD by the downregulation of miRNA-181b. Brain Res Bull. 2018;140:392-401. 